\title{
Harmonic expectation and affect in Western music: Effects of attention and training
}

\author{
Psyche Loui ANd David Wessel \\ University of California, Berkeley, California
}

\begin{abstract}
We investigated the effects of selective attention and musical training on the processing of harmonic expectations. In Experiment 1, participants with and without musical training were required to respond to the contour of melodies as they were presented with chord progressions that were highly expected, slightly unexpected, or extremely unexpected. Reaction time and accuracy results showed that when attention was focused on the melody, musically trained participants were still sensitive to different harmonic expectations, whereas participants with no musical training were undifferentiated across expectation conditions. In Experiment 2, participants were required to listen holistically to the entire chord progression and to rate their preference for each chord progression. Results from preference ratings showed that all the participants, with or without musical training, were sensitive to manipulations of harmonic expectations. Experiments 3 and 4 showed that changing the speed of presentation of chord progressions did not affect the pattern of results. The four experiments together highlight the importance of attentional focus in musical training, especially as it relates to the processing of harmonic expectations.
\end{abstract}

Music is a series of purposeful sounds; it is a temporal art, since time is necessary for the series of sounds to unfold. As time elapses while music is listened to, human listeners necessarily generate expectations for what will occur next in melody, rhythm, timbre, and harmony - the fundamental aspects of music.

Whereas melody is defined by the relationship between pitches presented sequentially over time, harmony typically concerns series of chords presented over time. Chords are groups of simultaneously presented pitches, and the psychological anticipation of progressive patterns of chords forms the basis of harmonic expectation. The formation and violation of harmonic expectations have been empirically tested and measured in many paradigms (e.g., Bharucha \& Stoeckig, 1986; Koelsch, Gunter, Friederici, \& Schröger, 2000; Krumhansl, 1990; Krumhansl \& Kessler, 1982; Schmuckler \& Boltz, 1994); such expectations have been shown to be present in individuals with and without formal musical training (Bigand, McAdams, \& Forêt, 2000; Koelsch, Schröger, \& Gunter, 2002) and have been postulated as the basis of the human affective response to music (Meyer, 1956).

Implicit indices for the fulfillment and violation of harmonic expectations have been shown using reaction time (RT; e.g., Bharucha \& Stoeckig, 1986; Bigand, Madurell, Tillmann, \& Pineau, 1999) and electrophysiology (Besson \& Faïta, 1995; Koelsch et al., 2000). In RT studies, participants have been given such tasks as consonance and dissonance judgments (Bharucha \& Stoeckig, 1986; Bigand et al., 1999), the detection of temporal asynchronies (Tillmann \& Bharucha, 2002), phoneme discrimina- tion (Bigand, Tillmann, Poulin, D'Adamo, \& Madurell, 1997), lexical decision (Poulin-Charronnat, Bigand, Madurell, \& Peereman, 2005), or timbre judgment (Tillmann, Bigand, Escoffier, \& Lalitte, 2006), and their expectations for harmony were manipulated using the principles of common practice music theory. Studies have repeatedly shown that participants are slower to respond to tonally unrelated chords. These results have been shown in both musicians and nonmusicians, suggesting that musical harmony can be processed implicitly and is independent of musical training.

Electrophysiological studies have confirmed the implicit formation of harmonic expectations. While listening to chord progressions that either fulfilled or violated harmonic expectations, participants with and without musical training showed several event-related potential (ERP) components in response to the violation of harmonic expectations. These ERP components include the early right anterior negativity (ERAN) and the N5. The ERAN is a negative deflection typically observed around $150-210 \mathrm{msec}$ after the expectancy-violating chord (Koelsch et al., 2000), and the N5 was a negative waveform 400-600 msec after the unexpected chord (Koelsch et al., 2000). These lines of research provide converging evidence that harmonic expectation is a reliable and quantifiable phenomenon in humans who have been exposed to Western music.

\section{Given a Melody, What Harmonies Do You \\ Expect? A Problem of Attention}

Although many studies have investigated expectations for either harmony or melody, relatively little work has

P. Loui, psyche@berkeley.edu 
addressed the interaction of these two musical attributes. Although robust effects have been observed in studies of harmonic expectation in participants with and without musical training (e.g., Bharucha \& Stoeckig, 1986; Justus \& Bharucha, 2001; Krumhansl \& Kessler, 1982; Schmuckler \& Boltz, 1994), few studies have investigated the formation of harmonic expectation during melodic perception. This is a particularly important issue in music perception because much of harmony in the classical music literature is not explicitly defined by chord progressions but could be implied using the pitches of the melody or an accompaniment to a melody. Without using chord progressions, these musical forms should still elicit similar harmonic expectations.

From the perspective of cognitive psychology, the formation of harmonic expectation during melodic perception is also important as a study in attention. Listening to music could be conceived as an exercise in divided attention among different musical attributes (Bigand et al., 2000; Jones, 2001; Jones \& Boltz, 1989), where harmony, melody, rhythm, and timbre are allocated cognitive resources in the formation of a unified musical percept. By measuring melodic perception during different conditions of harmonic expectation, we can approach the problem of music listening from the perspective of the interaction between allocated attentional resources. According to this view, if the psychological representations of melody and harmony were independent, performance on melodic perception should be independent of harmonic manipulations. Conversely, if our perception of melody is influenced by concurrently formed harmonic expectations, the violation of harmonic expectations should disrupt melodic perception.

In addition to the interaction of melodic and harmonic expectations, we are also interested in the effects of musical training on attention and music perception. Differences between musicians and nonmusicians have been observed in brain morphology (Gaser \& Schlaug, 2003), RT studies (Bigand et al., 1999; Tillmann \& Bharucha, 2002), and electrophysiological research (Besson \& Faïta, 1995; Koelsch, Schmidt, \& Kansok, 2002). A possible mediator of these differences involves attentional modulation: It is possible that musical training affects the way in which humans attend to music and form expectations.

The present study investigates the effects of attention on harmonic expectation by imposing an attentional constraint on musician and nonmusician participants during the study. In the first experiment, participants were instructed to listen only to the melody formed by the chords, making responses to the contour movement of the melody. Their RTs and accuracies were collected as different chord progressions were presented with these melodies. Chord progressions were in one of three conditions: high expectation, medium expectation, and low expectation. The participants were not given any instructions regarding the expectation conditions; they were told only to listen selectively to the melody, which remained the same across conditions. Using this method, we were able to investigate harmonic expectation during selective attention to melody.

\section{EXPERIMENT 1}

\section{Method}

Participants. Thirty-two students from the University of California at Berkeley participated in this study. All participants reported having normal hearing. Participants volunteered in this study in return for credit in undergraduate psychology courses.

The participants joined the study by enrolling in one of two groups. In the nonmusician group, the participants could enroll only if they reported having no musical training outside of normal school education. In the musician group, the participants could enroll only if they reported having had 5 or more years of musical training outside of normal schooling. Sixteen students volunteered in each group. The mean ages of the participants were 20.2 years (range, 18-23) in the musician group and 20.5 years (range, 18-25) in the nonmusician group. The musician group included 5 males and 11 females; the nonmusician group included 9 males and 7 females. The participants in the musician group had an average of 11.2 years of musical training (range, 6-17) in one or more instruments, including piano (12), violin (6), flute (3), clarinet (3), saxophone (2), and violoncello (1). None of the participants reported having absolute pitch.

Stimuli and Materials. The experiment was run in a soundproof chamber using an IBM laptop computer and AKG-K301 headphones. Software written in Max/MSP (Zicarelli, 1998) was used to generate all the stimuli, as well as to present the experiment and record responses. Auditory stimuli consisted of 30 chord progressions generated from sine tones. Each chord progression consisted of five chords presented at the rate of $2 \mathrm{sec}$ per chord, followed by a 2 -sec silence after the fifth chord. The relatively long interonset interval (IOI) was chosen so as to ensure that the participants had ample time to respond before the onset of the next chord. Each chord was a collection of four simultaneously presented pure tones, which gave rise to a sense of four-part harmony. For this experiment, melody was defined as the sequence of pitches formed by the highest pitch of each chord in the progression. Thus, each chord progression had a five-note melody, with one chord accompanying each note of the melody (see Figures 1 and 2).

For each melody, three chord progressions were constructed. The first type of chord progression was the high-expectation condition, which followed the music-theoretical rules of traditional tonal harmony; in Roman numeral notation, the chords were $\mathrm{I}-\mathrm{I}^{6}-\mathrm{IV}-\mathrm{V}-\mathrm{I}$. The second type of chord progression was $\mathrm{I}-\mathrm{I}^{6}-\mathrm{N}^{6}-\mathrm{V}-\mathrm{I}$, containing the unusual but compositionally valid Neapolitan chord in the third position; this progression was the medium-expectation condition. The third type was a low-expectation condition, with the progression $\mathrm{I}-\mathrm{I}^{6}-\mathrm{IV}-\mathrm{V}-\mathrm{N}^{6}$, in which the fifth chord was out of context and compositionally unacceptable according to common practice tonal music theory (see Figure 1). Ten chord progressions were generated for each condition; each progression was in the key of C major, D major, or E major; the progressions contained different voiceleading patterns, but all the progressions obeyed the traditional rules of voice leading in music theory (Piston, DeVoto, \& Jannery, 1987). Importantly, the same melodies occurred for all three conditions; that is, each melody was accompanied by one chord progression in the high-, medium-, and low-expectation conditions, respectively.

Procedure. The participants were presented with randomly ordered trials, each trial consisting of a five-chord progression. As each chord played, the participants' task was to respond as to whether the melody - defined here as the pitch sequence formed by the highest pitch of each of the successive chords - went up, went down, or stayed the same, as compared with the melodic pitch of the chord before it (see Figure 2). Thus, the temporal order of a trial was as follows. When the first chord was presented, the participants listened to the chord and were required to attend to its highest pitch. When the second chord was heard ( $2 \mathrm{sec}$ after the onset of the first chord), the participants made a speeded buttonpress indicating whether the second melodic pitch was higher, lower, or the same, as compared with the first. Then, when the third chord was heard, the participants made a similar response comparing the third melodic pitch with 
A

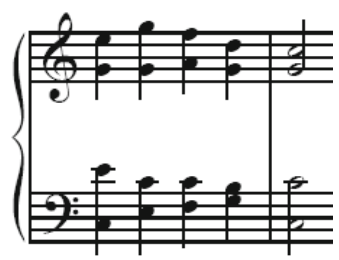

High Expectation I-I $\left.\right|^{6}-$ IV-V-I

B

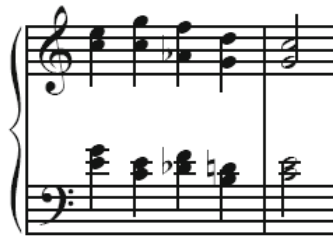

C

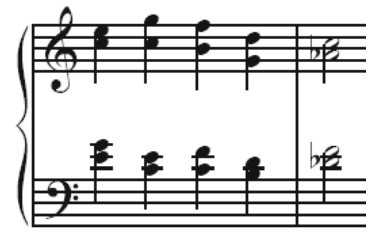

Medium Expectation $I-I^{6}-N^{6}-V-I$

Low Expectation $1-\left.\right|^{6}-\mid \mathrm{V}-\mathrm{V}-\mathrm{N}^{6}$

Figure 1. Examples of stimuli in musical notation. (A) Highexpectation condition. (B) Medium-expectation condition. (C) Low-expectation condition.

the second. The fourth melodic pitch was then compared with the third, and the fifth pitch was compared with the fourth. After the fifth chord, a 2-sec silent intertrial interval was presented. Nothing was mentioned to the participant about the harmonic structure of the chord progressions. The responses (up, down, or same) and RTs were collected and analyzed.

\section{Results}

For the musically trained participants, RTs increased as expectation decreased. This was confirmed by ANOVAs showing that in response to the final chords, average RT was significantly longer in the low-expectation condition than in the high-expectation condition [low expectation, $M=1,023 \mathrm{msec}, S D=340 \mathrm{msec}$; high expectation, $M=872 \mathrm{msec}, S D=346 \mathrm{msec} ; F(1,15)=18.001$, $p<.001]$, and RT for chords in the third position was significantly longer in the medium-expectation condition than in the high-expectation condition [medium expecta-

tion, $M=856 \mathrm{msec}, S D=267 \mathrm{msec}$; high expectation, $M=791 \mathrm{msec}, S D=270 \mathrm{msec} ; F(1,15)=5.619, p<$ $.02]$. This trend was not observed in musically untrained participants, who were slower to respond overall in both third-position chords [high expectation, $M=897 \mathrm{msec}$, $S D=348 \mathrm{msec}$; medium expectation, $M=839 \mathrm{msec}$, $S D=162 \mathrm{msec}$; overall $M=877 \mathrm{msec}, S D=340 \mathrm{msec}$; $F(1,15)=3.159$, n.s.] and final-position chords [high expectation, $M=927 \mathrm{msec}, S D=381 \mathrm{msec}$; low expectation, $M=898 \mathrm{msec}, S D=349 \mathrm{msec}$; overall $M=$ $917 \mathrm{msec}, S D=349 \mathrm{msec} ; F(1,15)<1$, n.s.] but were undifferentiated in average RT across all the expectation conditions [third chords, $F(1,15)=3.159$, n.s.; final chords, $F(1,15)=0.676$, n.s.; see Figure $3 \mathrm{~A}$ ]. A two-way ANOVA with factors of expectation (high, medium, and low) and musical training (musicians vs. nonmusicians) for the two dependent variables of RT in response to the third and fifth chords confirmed that musicians were faster than nonmusicians overall in response to chords in the third position $[F(1,30)=4.19, p<.05]$ but that the two groups were not significantly different in their RT for the fifth-position chords $[F(1,30)=1.90$, n.s. $]$. Importantly, there was a significant interaction between musical training and harmonic expectation in RTs for both third-position and fifth-position chords [third position, $F(1,30)=8.12, p<.01$; fifth position, $F(1,30)=12.6$, $p<.01]$.

As is shown in Figure 3B, accuracy was significantly above chance in all the conditions (chance performance $=$ $33 \%$ ). A two-way ANOVA with factors of expectancy and musical training confirmed that the musicians performed significantly better than the nonmusicians in both the third chords [musicians' mean accuracy $=92 \%, S D=$ $25 \%$; nonmusicians' mean accuracy $=77 \%, S D=41 \%$; $F(1,15)=40.504, p<.001]$ and the final chords [musicians' mean $=76 \%, S D=42 \%$; nonmusicians' mean $=$ $63 \%, S D=48 \% ; F(1,15)=11.547, p=.001]$. In response to the final chord, the musicians' accuracy was significantly lower in the low-expectation condition than in the high-expectation condition [low expectation, $M=$ $80 \%, S D=40 \%$; high expectation, $M=69 \%, S D=46 \%$; $F(1,15)=6.965, p<.01]$. In contrast, accuracy was unaffected by expectation condition in the nonmusicians [high

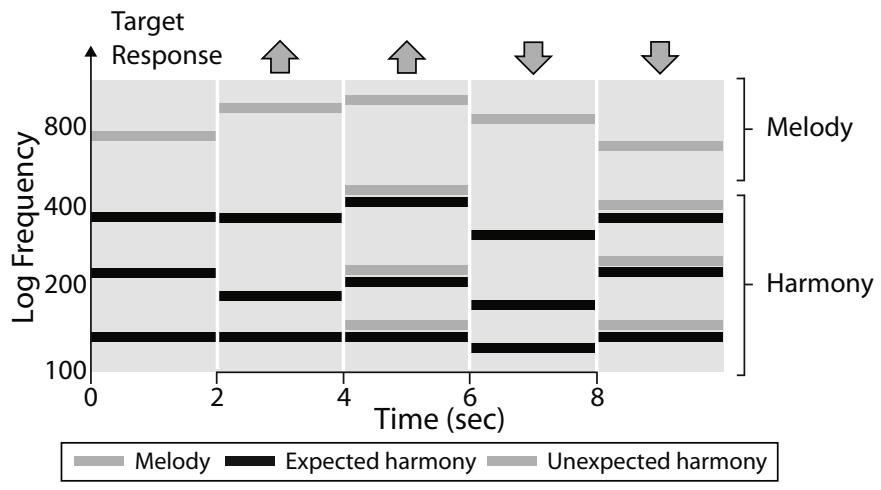

Figure 2. Examples of stimuli with target responses in pseudospectrogram representation. 

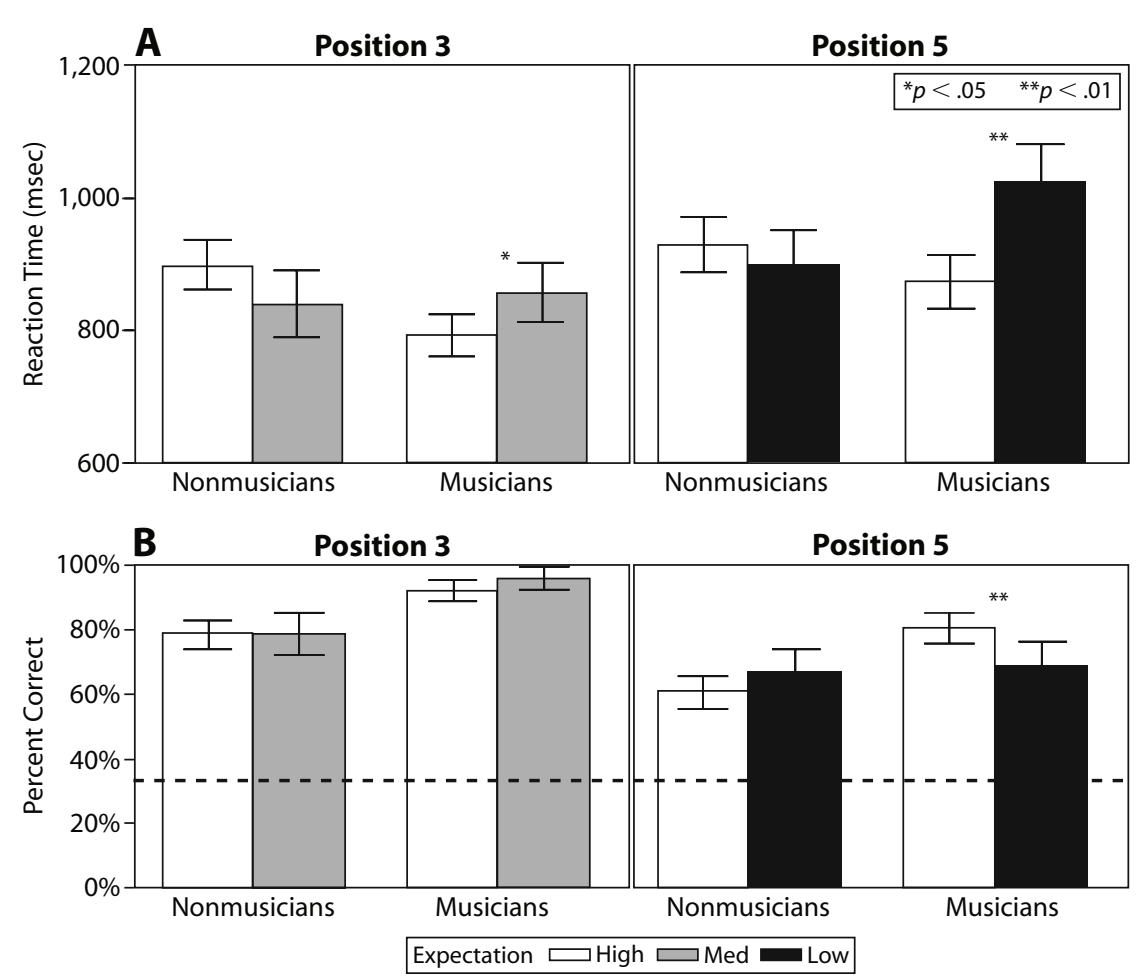

Figure 3. (A) Reaction time data from Experiment 1. (B) Accuracy data from Experiment 1. Error bars represent between-subjects standard errors. ${ }^{*} p<.05 .{ }^{* *} p<.01$.

expectation, $M=60 \%, S D=49 \%$; low expectation, $M=$ $67 \%, S D=47 \% ; F(1,15)=1.769$, n.s.]. For the third chord of the progressions, the nonmusicians were again undifferentiated across conditions [high expectation, $M=$ $78 \%, S D=41 \%$; low expectation, $M=78 \%, S D=41 \%$; $F(1,15)=0.000$, n.s.]. The musicians' performance was at ceiling levels in both high- and medium-expectation conditions (high expectation, $M=92 \%, S D=28 \%$; low expectation, $M=96 \%, S D=20 \%$ ) and did not differ significantly between conditions $[F(1,15)=2.449$, n.s. $]$. In conclusion, no speed-accuracy trade-off was observed between RT and accuracy for both the musicians and the nonmusicians. RTs and accuracy for chords in other positions were undifferentiated across expectancy conditions for both groups.

\section{Discussion}

The experiment above showed that musically trained participants were faster at responding to melodies when chords accompanying these melodies were harmonically expected. This facilitated RT could not be attributed to a speed-accuracy trade-off, since shorter RTs were accompanied by similar or higher accuracy levels in both groups of participants. When the melodies were accompanied by unexpected chords, the musicians' performance was impaired, whereas the nonmusicians were unaffected. These results suggest that harmonic expectations affect melodic perception only in individuals with musical training.

Although these differences in performance on the task between the musicians and the nonmusicians could be at- tributed to differences in attentional resources, it could be the case that the nonmusicians were insensitive to the manipulation of harmonic expectation simply because they could not hear the differences between different chords. To investigate this possibility, we varied the task in another experiment so that although the participants were presented with the same chord progressions, the demand of selective attention was removed, so that the participants listened holistically to the chord progressions. In order to investigate the effects of expectation violations on the human affective response to music, we presented the participants with chord progressions similar to those in Experiment 1. The participants' task was to listen to the entire chord progression and then to rate the chord progressions on the basis of their preferences.

\section{EXPERIMENT 2}

The second experiment employed the simple method of subjective preference ratings for chord progressions. Chord progressions were the same as those in the previous experiment, and the participants were the same students as those who had volunteered for the previous experiment. By obtaining ratings from musically trained and untrained participants for chord progressions of high, medium, and low expectation, we hoped to answer the following questions: (1) How do harmonic expectations influence preferences? (2) How do ratings compare across musical training? (3) How does the removal of an attentional demand change the behavior of musicians and nonmusi- 
cians? Using a simple 4-point rating system, the present experiment attempted to relate affective arousal in music to expectation within a Western harmonic framework.

\section{Method}

Participants. The same 32 individuals as those in Experiment 1 participated in Experiment 2.

Stimuli and Materials. All the materials used in this experiment were the same as those in Experiment 1. Chord progressions were the same as those in Experiment 1, except for one modification: Chord progressions were played at a rate of $0.8 \mathrm{sec}$ per chord in this experiment, with an intertrial interval of $0.8 \mathrm{sec}$. This was a faster presentation rate than that in Experiment 1, in which the chord progressions were presented at $2 \mathrm{sec}$ per chord. This modification was due to the different experimental design: Experiment 2 required that the participants listen to the entire chord progression before responding, whereas Experiment 1 required responding at every chord. The chord progressions were played at $0.8 \mathrm{sec}$ per chord in order to ensure that the participants remained alert during Experiment 2.

Procedure. In this experiment, the participants' task was to listen to the entire chord progression and then rate their preference for the chord progression on a scale of 1 (very unpleasant) to 4 (very pleasant). The participants responded by typing their numerical response on the keyboard of the laptop computer.

\section{Results}

The participants rated chord progressions in the highexpectation condition as most pleasant $(M=3.16, S D=$ $0.82)$, the medium-expectation progressions as less pleasant $(M=2.75, S D=0.84)$, and the low-expectation progressions as the least pleasant $(M=1.56, S D=0.72)$. These effects were robust, and the pattern of results did not vary significantly between musically trained and untrained participants. The effect of expectation condition was confirmed as significant by a one-way repeated measures ANOVA $[F(2,45)=385.1, p<.0001]$. The musicians' ratings were generally higher than the nonmusicians' ratings $[F(1,30)=14.31, p<.0001]$, but there was no significant interaction between expectation condition and musical training $[F(2,90)=0.026, p=.974$, n.s.; see Figure 4].

\section{Discussion}

Experiment 2 showed that when asked to attend holistically to the chord progressions, both the musicians and

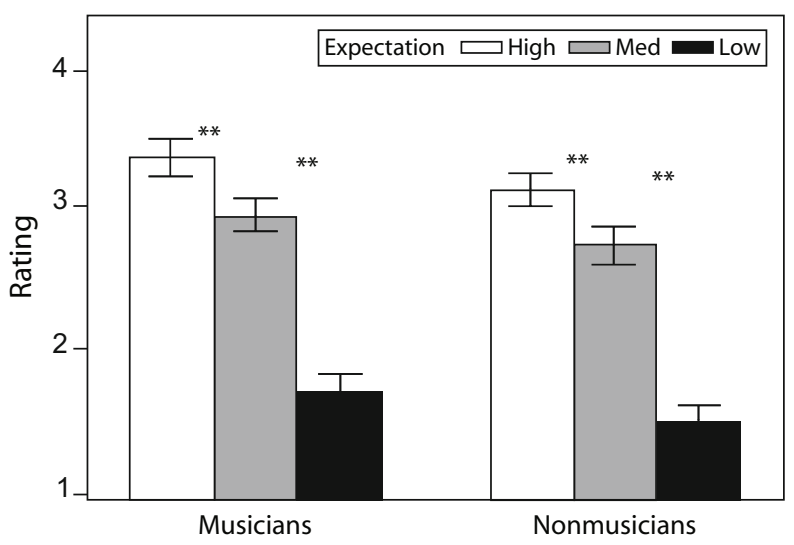

Figure 4. Preference ratings in Experiment 2. Error bars represent between-subjects standard errors. ${ }^{* *} p<.01$. the nonmusicians could perceive the difference between different expectation conditions, as is evident in their preference ratings. The parallelism between the musicians' and the nonmusicians' results is in contrast with data from Experiment 1, which showed significant effects of harmonic expectation in musicians, but not in nonmusicians, resulting in a significant interaction between expectation and musical training.

Several hypotheses could account for the results observed in Experiments 1 and 2. One possible interpretation is that musical training strengthens the automatic formation of harmonic expectations. Results from a comparison between Experiment 1 (attention-demanding task) and Experiment 2 (with a task less demanding of attention) are consistent with the view that musically trained participants form harmonic expectations independently of attention, whereas attention is required for the modulation of harmonic expectation in nonmusicians. It is possible that during music lessons, melodies are always presented with harmonically expected chords, leading to an implicit coupling of melodic pitches with their expected harmonies. This implicit coupling is usually helpful, since melodies are usually accompanied by harmonically expected chord progressions. However, automatically formed harmonic expectations can harm performance when the melody is accompanied by an unexpected harmony. In this case, musicians will be unable to ignore the distracting harmony, whereas nonmusicians who are not paying attention to the harmony will be unaffected by harmonic changes. This theoretical account would predict facilitated performance in expected harmonies and inhibited performance in unexpected harmonies in musicians, as well as no effect of harmonic expectation on musically untrained individuals.

If the attention-dependent view of expectation formation is correct, expectation formation should be the same for musicians and nonmusicians under conditions of maximal attentional resources (with no cost of attention). Thus, when listening holistically to chord progressions, musicians and nonmusicians should form the same harmonic expectations. In Experiment 2, we removed any demands of selective attention by encouraging the participants to listen holistically to the chord progressions. Experiment 2 showed that the participants preferred highly expected chord progressions and strongly disliked chord progressions that violated expectations. Both the musicians and the nonmusicians were able to distinguish the three types of harmonic expectations while attending holistically to the chord progressions in the preference-ratings task. The pattern of results across the three expectation conditions was the same, regardless of musical training.

Although the differential effects of Experiments 1 and 2 were probably due to the differential selective attention manipulations, a possible alternate interpretation is that the interaction between the musicians and the nonmusicians' results in Experiment 1 were due to differential working memory loads for the musicians and the nonmusicians. Since the IOI between successive chords in Experiment 1 was set to the slow tempo of $2 \mathrm{sec}$, it is possible that the chord progressions as a whole exceeded the 
nonmusicians' working memory load, whereas the musicians' working memory load was sufficiently large to show sensitivity to expectancy manipulations of the chord progressions. This hypothesis is supported by the observation that the harmonic priming effect starts to diminish at the tempo of $2.5 \mathrm{sec}$ per chord (Tekman \& Bharucha, 1992). To investigate the competing hypotheses of attention versus working memory as possible cognitive generators of the present results, we conducted Experiment 3, in which the time interval between successive chords was shortened so as to ensure that chord progressions were within the limit of short-term memory for both musicians and nonmusicians.

\section{EXPERIMENT 3}

\section{Method}

Participants. Thirty-two undergraduates from the University of California at Berkeley participated in this experiment in return for course credit. All the participants had normal hearing. Sixteen of the participants were recruited on the basis of having 5 or more years of musical training, whereas the remaining 16 participants were recruited on the basis of having no musical training. Musically trained participants ( 11 females, 5 males; average age, 20 years; age range, 18-25) had an average of 10 years of musical training (range, 5-18) in piano (14), cello (2), flute (5), clarinet (1), trombone (1), and percussion (2). None of the nonmusician group (10 females, 6 males; average age, 20 years; range, 18-27) had any musical training outside of normal school education.

Stimuli and Materials. Stimuli, materials, procedures, and the participants' task were the same as those in Experiment 1, except for the time delay between successive chords in the chord progression. For all the chord progressions, the IOI between successive chords in each progression was $1,200 \mathrm{msec}$.

\section{Results}

For the musicians, RT in response to the third chord was significantly shorter in the high-expectation condition $(M=665 \mathrm{msec}, S D=126 \mathrm{msec})$ than in the mediumexpectation condition $(M=701 \mathrm{msec}, S D=111 \mathrm{msec})$. This was confirmed by a repeated measures ANOVA comparing average RT in the two conditions $[F(1,15)=$ $4.75, p<.05]$. RT in response to the fifth chord was significantly shorter in the high-expectation condition $(M=$ $674 \mathrm{msec}, S D=126 \mathrm{msec}$ ) than in the low-expectation condition $(M=791 \mathrm{msec}, S D=144 \mathrm{msec})$, as confirmed by a repeated measures ANOVA comparing the two RTs $[F(1,15)=21.5, p<.001]$. Thus, the results demonstrate that musically trained participants were sensitive to the manipulation of harmonic expectation.

The nonmusicians showed no significant differences between all three expectation conditions. In the third position, RT for the high-expectation condition $(M=$ $748 \mathrm{msec}, S D=154 \mathrm{msec}$ ) was not significantly different from that for the medium-expectation condition $(M=$ $802 \mathrm{msec}, S D=118 \mathrm{msec}$ ) $[t(10)=1.55$, n.s.], and in the fifth position, RT for the high-expectation condition ( $M=$ $774 \mathrm{msec}, S D=117 \mathrm{msec}$ ) was not significantly different from that for the low-expectation condition $(M=$ $748 \mathrm{msec}, S D=119 \mathrm{msec}$ ), suggesting that the participants without formal musical training were not affected by manipulations of harmonic expectation during the melodic contour judgment task.

A comparison between the musicians and the nonmusicians showed significantly shorter RTs for the musicians overall $[F(1,90)=4.98, p<.05]$. Importantly, for RT in response to the fifth chord, there was a significant interaction between musical training and expectation condition $[F(1,90)=3.53, p<.05]$, confirming that the musicians were more sensitive to the manipulation of harmonic expectation, as compared with nonmusicians (see Figure 5A).

Accuracy data, plotted as percent correct in the melodic contour identification task (Figure 5B), showed no significant differences across all the conditions for both the musicians and the nonmusicians. Thus, there was no speedaccuracy trade-off between RT and accuracy results. The musicians, however, were generally more accurate than the nonmusicians, as revealed by a main effect of musical training [one-way ANOVA, $F(1,126)=45.6, p<.001$ ] RTs and accuracy for chords in other positions were undifferentiated across expectancy conditions for both groups.

\section{Discussion}

Experiment 3 showed that when the task was to judge the contour of melodies, musicians were still affected by the manipulation of harmonic expectation, whereas nonmusicians were insensitive to these manipulations. Using a shorter IOI, we observed results in Experiment 3 similar to those obtained in Experiment 1. This eliminates the hypothesis that the different levels of sensitivity to harmonic expectation in the musicians and the nonmusicians were due to differences in working memory load.

To ensure that the IOI of $1.2 \mathrm{sec}$ would still elicit the same emotional responses in both musicians and nonmusicians, we replicated Experiment 2 with the same IOI as that used in Experiment 3.

\section{EXPERIMENT 4}

\section{Method}

Participants. The same participants as those in Experiment 3 also took part in Experiment 4.

Stimuli and Materials. Stimuli, materials, and procedures were the same as those in Experiment 2, except for the time delay between successive chords in the chord progressions. For all chord progressions, the IOI between successive chords in each progression was $1,200 \mathrm{msec}$.

\section{Results}

The participants rated chord progressions in the highexpectation condition as most pleasant $(M=3.27, S D=$ $0.49)$, the medium-expectation progressions as slightly less pleasant $(M=2.92, S D=0.36)$, and the low-expectation progressions as the least pleasant $(M=1.83, S D=0.48)$. The pattern of results did not vary significantly between musically trained and untrained participants. The effect of expectation condition was confirmed as significant by a two-way ANOVA with factors of expectation and musical training $[F(2,90)=97.9, p<.0001]$. The musicians' ratings were generally higher than the nonmusicians' ratings $[F(1,90)=4.78, p<.05]$, but there was no interac- 

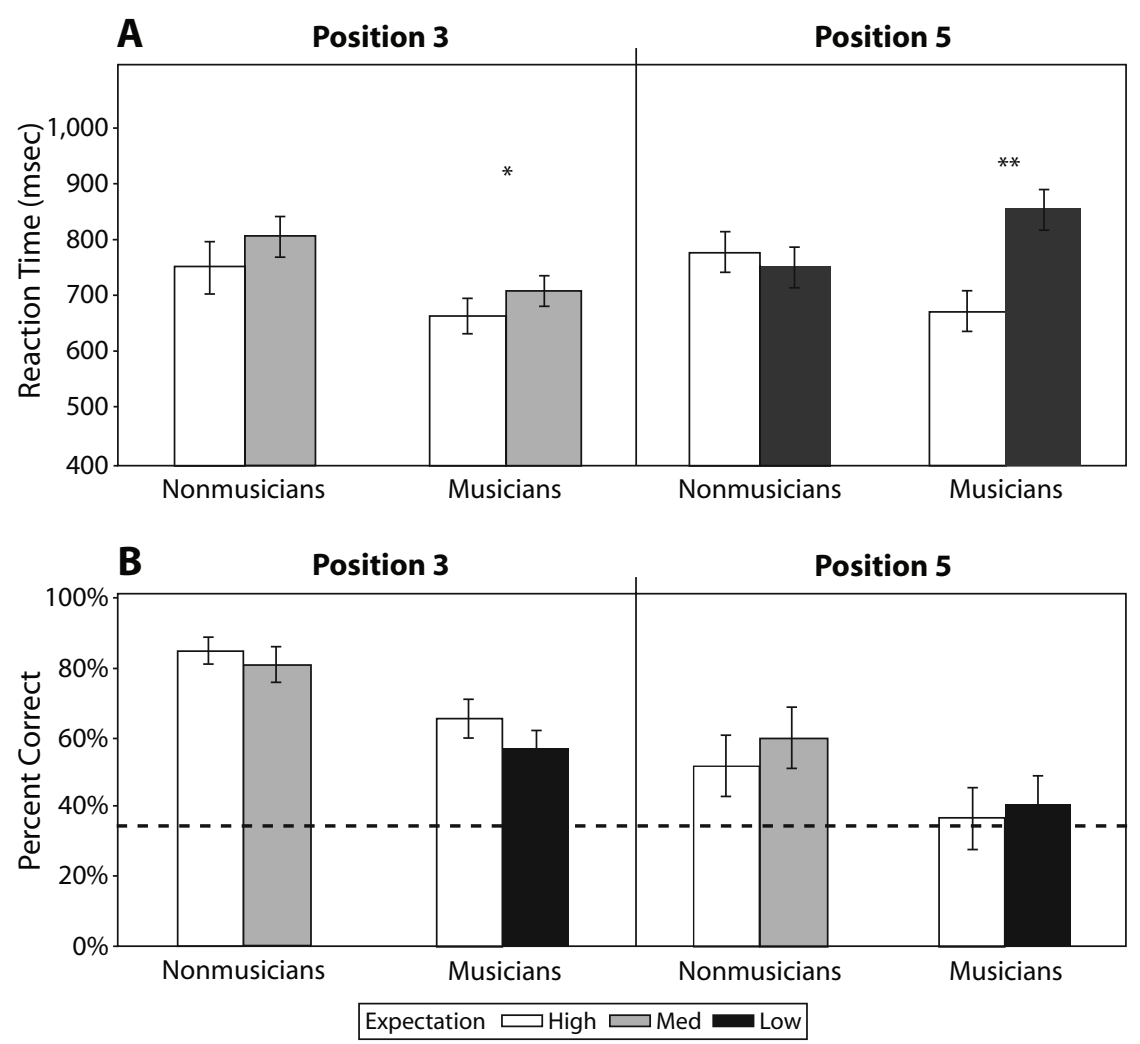

Figure 5. (A) Reaction time data from Experiment 3. (B) Accuracy data from Experiment 3. Error bars represent between-subjects standard errors. ${ }^{*} p<.05 .{ }^{* *} p<.01$.

tion between expectation condition and musical training $[F(2,90)=2.69$, n.s.; see Figure 6].

\section{Discussion}

Using chord progressions with a tempo (IOI) similar to that in Experiment 3, Experiment 4 replicated the results from Experiment 2 in confirming that both musically trained and untrained participants could perceive the differences between harmonic expectation conditions when

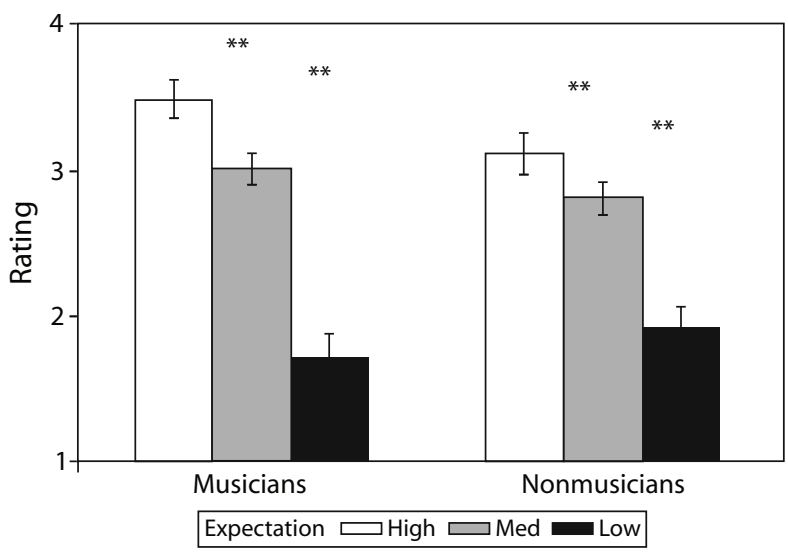

Figure 6. Preference ratings in Experiment 4. Error bars represent between-subjects standard errors. ${ }^{* *} p<.01$. asked to attend holistically to the chord progressions. As in Experiment 2, the most highly expected chord progressions were most highly preferred, and changing the tempo of chord progressions did not change the pattern of results.

\section{GENERAL DISCUSSION}

The results from the present experiments showed that when given a cost of attention, musically trained individuals were sensitive to harmonic expectation, whereas musically untrained individuals were unaffected by different harmonies. The dissociation between data from musicians and nonmusicians was clear during the melodic contour perception task, which imposed an attention-demanding task over the manipulation of harmonic expectation. When the demand of selective attention was removed, however, musicians and nonmusicians were similarly affected by harmonic expectation, as was shown in the subjective ratings task, which allowed the participants to listen holistically to the entire chord progressions. The results could not be attributed to differential demands in working memory for musicians and nonmusicians, since speeding up successive chords in the chord progression (Experiment 3 ) did not change the patterns of results. Because of the manipulation of selective attention, the melodic contour judgment task in Experiments 1 and 3 showed interactions between musical training and harmonic expectation, 
where musicians were sensitive to harmonic expectation even during a melodic contour judgment task, whereas nonmusicians were insensitive to the manipulation of harmonic expectation. Removing the attentional demand in Experiments 2 and 4 resulted in similar effects, regardless of musical training. These results are consistent with a model of attention in which musical training selectively facilitates responses to harmonically expected chord progressions, so that musicians form automatic expectations for prototypical chord progressions and are unable to ignore these harmonic expectations even when given an orthogonal task.

The automatic expectation formation model also fits with data from electrophysiological studies (Koelsch, Schröger, \& Gunter, 2002) in which the ERAN, an ERP component elicited by the violation of harmonic expectation, was compared between musicians and nonmusicians. The results of the study showed that although the ERAN was significantly elicited in both musicians and nonmusicians, a larger ERAN was observed in musicians than in nonmusicians, suggesting that expectation is formed automatically in musicians. Another study showed that the violation of harmonic expectation is heavily modulated by attention in nonmusicians (Loui, Grent-’t-Jong, Torpey, \& Woldorff, 2005). Taken together, these results suggest that the formation of harmonic expectation in nonmusicians is sensitive to attentional modulation, whereas expectation for harmony may be automatic in musicians. These results are consistent with the present study in showing that given an attention-demanding task, musicians are still sensitive to violations of harmonic expectations, whereas nonmusicians are insensitive to changes in harmony, due to the allocation of attentional resources to task performance.

On the basis of these results, we conclude that attention strongly modulates the formation of harmonic expectation in nonmusicians but does so to a lesser degree in musicians. Harmonic expectation may be relatively automatically formed in persons with musical training, so that when given a related but attention-demanding task, musically trained participants are still affected by harmonic expectation. The present results differ from previous results that have shown no interaction between harmonic expectations and musical training (e.g., Bigand et al., 1999). These divergent results may be due to the nature of the different tasks: In our study, the participants were required to listen selectively for the melody, whereas previous studies required holistic processing of the chords. The striking difference between our results and those in previous studies suggests that attention is an important factor in music processing.

The results from Experiment 2 showed that affective ratings for musical chord progressions decreased as harmonic expectation was violated. These results converge with data from Steinbeis, Koelsch, and Sloboda (2006), who observed increases in tension ratings and physiological arousal as a function of harmonic expectation. However, these results are only partially in support of Meyer's (1956) postulated inverse-U-curve relationship between expectation and affective arousal. According to Meyer, music that slightly deviated from expectation would be experienced as most pleasurable and most emotionally arousing, whereas music that was highly expected and highly unexpected would be less interesting or arousing. From our data, the most unexpected harmonic progressions were rated as least pleasurable. This point coincides with Meyer's predictions; however, contrary to Meyer's model, highly expected harmonic progressions were rated as most pleasurable. Several factors could account for this discrepancy between our data and Meyer's prediction. First of all, Meyer was referring to the violation of expectation as it relates to each complex case in real composed music. In contrast, the present study investigated the effects of fixed, repeated, and simple harmonic manipulations on pleasantness ratings; thus, Meyer's predictions may be inapplicable in the present experimental conditions. Second, Meyer's discussion of expectation and expectancy violation could be seen as operating on a different scale from the scope of the present study. Meyer discusses musical expectation as arising from repeated cultural exposure to various elements of Western music, including melodic contour, rhythmic grouping, and stylistic norms. Our study, as is true for any controlled study, attempted to manipulate only one element: harmonic expectation. Perhaps the manipulation of only one variable in music is not enough to generate an emotional response in the hypothesized direction. Our perception of affect in music may be the result of complex interactions among the musical attributes of melody, rhythm, and timbre and the cultural background and cognitive constraints of each individual listener. Nevertheless, from the present results, we conclude that within the experimental environment, human listeners strongly prefer chord progressions that fulfill their expectations and that this preference is unaffected by musical training.

In Experiment 3, the IOI between successive chords was decreased to investigate the possibility that the differences observed between musicians and nonmusicians were due to differential working memory constraints. Prior research in musical tempo (e.g., Clarke, 1999) has shown that memory for musical sounds diminishes at the slow presentation rate of $2 \mathrm{sec}$. If the difference between musicians and nonmusicians were due to differential memory constraints, a faster presentation rate would lead to decreased memory constraints and would thus equate results between the two groups. However, the results from Experiment 3 converge with the data from Experiment 1, showing that during an attention-demanding melodic contour judgment task, musically trained participants were still sensitive to the influence of harmony, whereas musically untrained participants were unaffected by the manipulation of harmonic expectation. By shortening the IOI to be within the constraints of memory, the parallel patterns of results between Experiment 3 and Experiment 1 suggest that the differences observed in musically trained and untrained participants were not due to differential memory constraints but to the attentional demands of the melodic contour judgment task. One of the results of longterm musical training may be to form automatic expectations for typical harmonies, so that musicians processed harmonies preattentively, regardless of their relevance to 
the task. Nonmusicians, on the other hand, were able to focus their attention exclusively on the melody due to task demands, while ignoring harmonies that were unrelated to the task.

Taken together, the results of the four experiments support the claim that attention and musical training differentially modulate the formation of harmonic expectations. When attention was selectively focused on the melody, participants' sensitivity to harmony depended on their musical training, but given no focus of selective attention, all participants became sensitive to harmonic expectations. The nature of the affective arousal associated with each type of chord progression, as well as the hypothesized role of expectation violation in evoking affect (Meyer, 1956), are factors that remain to be disentangled in future studies.

\section{AUTHOR NOTE}

This research was supported by a grant from the Academic Senate of the University of California at Berkeley to D.W. We thank E. Wu and Y. Lee for help with data collection, E. Hafter and W. Prinzmetal for helpful comments and the use of their laboratory space, and E. Bigand, C. Hudson Kam, and E. Wu for valuable suggestions on the manuscript. Correspondence concerning this article should be addressed to P. Loui, Center for New Music and Audio Technologies, University of California, 1750 Arch St., Berkeley, CA 94709 (e-mail: psyche@berkeley.edu).

\section{REFERENCES}

BESSON, M., \& FAÏTA, F. (1995). An event-related potential (ERP) study of musical expectancy: Comparison of musicians with nonmusicians. Journal of Experimental Psychology: Human Perception \& Performance, 21, 1278-1296.

Bharucha, J. J., \& Stoeckig, K. (1986). Reaction time and musical expectancy: Priming of chords. Journal of Experimental Psychology: Human Perception \& Performance, 12, 403-410.

Bigand, E., Madurell, F., Tillmann, B., \& Pineau, M. (1999). Effect of global structure and temporal organization on chord processing. Journal of Experimental Psychology: Human Perception \& Performance, 25, 184-197.

Bigand, E., McAdams, S., \& Forêt, S. (2000). Divided attention in music. International Journal of Psychology. 35, 270-278.

Bigand, E., Tillmann, B., Poulin, B., D’Adamo, D. A., \& Madurell, F. (1997). The effect of harmonic context on phoneme monitoring in vocal music. Cognition, 81, B11-B20.

Clarke, E. F. (1999). Rhythm and timing in music. In D. Deutsch (Ed.), The psychology of music (pp. 473-500). San Diego: Academic Press.

GaSer, C., \& SCHLAUg, G. (2003). Brain structures differ between musicians and non-musicians. Journal of Neuroscience, 23, 9240-9245.

Jones, M. R. (2001). Temporal expectancies, capture, and timing in auditory sequences. In C. L. Folk \& B. S. Gibson (Eds.), Attraction, distraction and action: Multiple perspectives on attentional capture (pp. 191-229). Amsterdam: Elsevier.

JoNES, M. R., \& BolTz, M. (1989). Dynamic attending and responses to time. Psychological Review, 96, 459-491.

Justus, T. C., \& BHARUCHA, J. J. (2001). Modularity in musical processing: The automaticity of harmonic priming. Journal of Experimental Psychology: Human Perception \& Performance, 27, 1000-1011.

Koelsch, S., Gunter, T. [C.], Friederici, A. D., \& Schröger, E. (2000). Brain indices of music processing: "Nonmusicians" are musical. Journal of Cognitive Neuroscience, 12, 520-541.

Koelsch, S., Schmidt, B.-H., \& KansoK, J. (2002). Effects of musical expertise on the early right anterior negativity: An event-related brain potential study. Psychophysiology, 39, 657-663.

Koelsch, S., Schröger, E., \& Gunter, T. C. (2002). Music matters: Preattentive musicality of the human brain. Psychophysiology, 39, $1-11$.

Krumhansl, C. L. (1990). Cognitive foundations of musical pitch. Oxford: Oxford University Press.

Krumhansl, C. L., \& Kessler, E. J. (1982). Tracing the dynamic changes in perceived tonal organization in a spatial representation of musical keys. Psychological Review, 89, 334-368.

Loui, P., Grent-'T-Jong, T., Torpey, D., \& WoldorfF, M. G. (2005). Effects of attention on the neural processing of harmonic syntax in Western music. Cognitive Brain Research, 25, 678-687.

Meyer, L. (1956). Emotion and meaning in music. Chicago: University of Chicago Press.

Piston, W., DeVoto, M., \& Jannery, A. (1987). Harmony (5th ed.). New York: Norton.

Poulin-Charronnat, B., Bigand, E., Madurell, F., \& Peereman, R. (2005). Musical structure modulates semantic priming in vocal music. Cognition, 94, B67-B78.

SChMUCKLeR, M. A., \& Boltz, M. G. (1994). Harmonic and rhythmic influences on musical expectancy. Perception \& Psychophysics, 56, 313-325.

Steinbeis, N., Koelsch, S., \& Sloboda, J. A. (2006). The role of harmonic expectancy violations in musical emotions: Evidence from subjective, physiological, and neural responses. Journal of Cognitive Neuroscience, 18, 1380-1393.

Tekman, H. G., \& Bharucha, J. J. (1992). Time course of chord priming. Perception \& Psychophysics, 51, 33-39.

Tillmann, B., \& Bharucha, J. J. (2002). Effect of harmonic relatedness on the detection of temporal asynchronies. Perception \& Psychophysics, 64, 640-649.

Tillmann, B., Bigand, E., Escoffier, N., \& Lalitte, P. (2006). The influence of musical relatedness on timbre discrimination. European Journal of Cognitive Psychology, 18, 343-358.

ZICARELLI, D. (1998). An extensible real-time signal processing environment for Max. In Proceedings of the International Computer Music Conference, 1998 (pp. 463-466). San Francisco: ICMA.

(Manuscript received December 29, 2005; revision accepted for publication February 27, 2007.) 